\title{
GRAVITATIONAL LENSING BY CURVED COSMIC STRINGS
}

\author{
MALCOLM R. ANDERSON \\ Department of Mathematics \\ Edith Cowan University \\ 2 Bradford St., Mt Lawley \\ WA 6050 Australia
}

\begin{abstract}
I briefly summarize all that is known about gravitational lensing by cosmic strings.
\end{abstract}

Cosmic strings are long thin filaments of Higgs field energy whose existence is predicted by a wide range of grand unified field theories (Vilenkin 1985). The earliest studies of cosmic string networks suggested that string loops could provide a simple and natural mechanism for the seeding of galaxy clusters (Zel'dovich 1980; Vilenkin 1981), but more recent numerical simulations indicate that networks have more small-scale power than first thought, leading to an excess of low-mass high-velocity loops which are unlikely to seed galaxy formation (Albrecht \& Turok 1989, Bennett \& Bouchet 1990).

Although cosmic strings have thus fallen into disfavor as cosmogonic agents, they may still have formed and played some role in the evolution of the early Universe. Apart from their characteristic Kaiser-Stebbins signature in the cosmic microwave background (Kaiser \& Stebbins 1984) the best chance of detecting them seems to be through gravitational lensing.

The type of lensing behavior expected in the neighborhood of a cosmic string can be straightforward or extremely complex, depending on the geometric configuration of the string involved. The simplest possible model is the infinite straight string (Gott 1985; Hiscock 1985) which produces two unmagnified and undistorted images of any source whose line-of-sight passes sufficiently close to the string. The separation of the two images is proportional to the mass per unit length of the string. For a GUT string, the images would typically be separated by a few arcseconds. 
However, numerical simulations suggest that strings have structure on all scales down to a characteristic radiative damping length. Realistic models need to take this small-scale structure into account. The first attempt to model lensing by string loops was made by Hogan and Narayan (1984), who assumed that the rays passing through the interior of a loop are undeflected while those passing outside it experience a spherical potential due to the mass of the string, plus a quadrupole moment. Although the model is very simplistic, it can give rise to one, two or three images, both magnified and unmagnified, plus caustics with often very complex geometry.

Unlike the metric outside a straight string - which is locally flat - the metric outside a curved horizon-sized string is characterized by the superposition of two distinct families of gravitational waves: traveling waves and curvature waves (Anderson 1995). Traveling waves, which are decoupled from the geometry of the string and can have arbitrary shape, were first discovered by Garfinkle (1990). Gravitational lensing by traveling waves has been examined numerically by Vollick and Unruh (1991), who found that they typically form double images whose separation varies as the wave components cross the line-of-sight.

Curvature waves are more interesting. Very difficult to treat mathematically, they are generated in response to high-curvature structure on the string. The only known exact solution containing curvature waves is the Aryal-Ford-Vilenkin (1986) metric, which describes a straight string passing through a Schwarzschild hole. It combines the lensing properties of the Schwarzschild metric and the straight string, and can produce up to four images. It is likely that the lensing effect of more general curvature waves would be similar to that of a series of black holes, each with mass proportional to the local curvature, threaded on a straight string. Their signature would thus be two pairs of two images, spaced symmetrically about the axis of the string, showing high variability in separation and magnitude.

\section{References}

Albrecht, A., \& Turok, N., 1989, Phys Rev D, 40, 973

Anderson, M.R., 1995, Aust J Phys, in press

Aryal, M., Ford, L.H., \& Vilenkin, A., 1986, Phys Rev D, 34, 2263

Bennett, D.P., \& Bouchet, F.R., 1990, Phys Rev D, 41, 2408

Garfinkle, D., 1990, Phys Rev D, 41, 1112

Gott, J.R., 1985, ApJ, 288, 422

Hiscock, W., 1985, Phys Rev D, 31, 3288

Hogan, C., \& Narayan, R., 1984, MNRAS, 211, 575

Kaiser, N., \& Stebbins, A., 1984, Nature, 310, 391

Vilenkin, A., 1981, Phys Rev Lett, 46, 1169

Vilenkin, A., 1985, Phys Rep, 121, 263

Vollick, D.N., \& Unruh, W.G., 1991, Phys Rev D, 44, 2388

Zel'dovich, Ya. B., 1980, MNRAS, 192, 663 